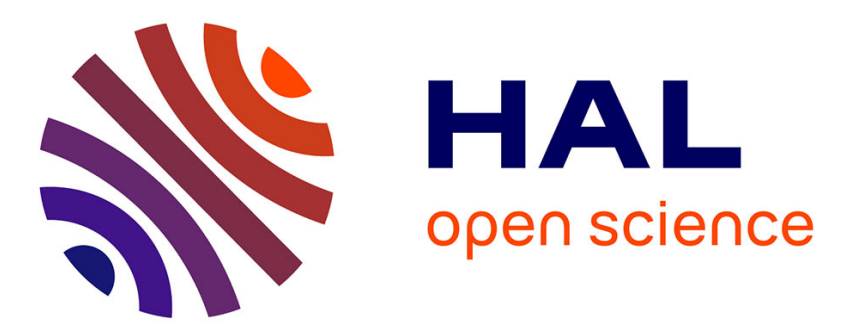

\title{
Parametric regression of 3D medical images through the exploration of non-parametric regression models
}

\author{
Christof Seiler, Xavier Pennec, Mauricio Antonio Reyes Aguirre
}

\section{To cite this version:}

Christof Seiler, Xavier Pennec, Mauricio Antonio Reyes Aguirre. Parametric regression of 3D medical images through the exploration of non-parametric regression models. Seventh IEEE International Symposium on Biomedical Imaging 2010 (ISBI'10), 2010, Rotterdam, Netherlands. pp.452-455, 10.1109/ISBI.2010.5490313 . inria-00616168

\section{HAL Id: inria-00616168 \\ https://hal.inria.fr/inria-00616168}

Submitted on 28 Jun 2013

HAL is a multi-disciplinary open access archive for the deposit and dissemination of scientific research documents, whether they are published or not. The documents may come from teaching and research institutions in France or abroad, or from public or private research centers.
L'archive ouverte pluridisciplinaire HAL, est destinée au dépôt et à la diffusion de documents scientifiques de niveau recherche, publiés ou non, émanant des établissements d'enseignement et de recherche français ou étrangers, des laboratoires publics ou privés. 


\title{
PARAMETRIC REGRESSION OF 3D MEDICAL IMAGES THROUGH THE EXPLORATION OF NON-PARAMETRIC REGRESSION MODELS
}

\author{
Christof Seiler $^{1}$, Xavier Pennec ${ }^{2}$ and Mauricio Reyes ${ }^{1}$ \\ ${ }^{1}$ University of Bern, ISTB, Bern, Switzerland \\ ${ }^{2}$ INRIA Sophia, Asclepios Team, Sophia Antipolis, France
}

\begin{abstract}
Currently there is an increase usage of CT-based bone diagnosis because low-radiation and cost-effective $2 \mathrm{D}$ imaging modalities do not provide the necessary 3D information for bone diagnosis. The fundamental objective of our work is to build a model connecting 2D X-ray information to 3D CT information through regression. As a first step we propose an univariate non-parametric regression on individual predictor variables to explore the non-linearity of the data. To later combine these univariate models we then replace them with parametric models. We examine two predictors, shaft length and caput collum diaphysis angle on a database of $182 \mathrm{CT}$ images of femurs. We show that for each predictor it is possible to describe $99 \%$ of the variance through a simple up to second order parametric model. These findings will allow us to extend to the multivariate case in the future.
\end{abstract}

Index Terms - Non-Parametric Regression, Parametric Regression, Log-Euclidean Framework, Diffeomorphic Deformations, Femur

\section{INTRODUCTION}

Up to now, 3D bone anatomy has been generated from X-ray images using computational tools. However these tools have focused only on 3D bone shape reconstruction while little attention has been given to $3 \mathrm{D}$ reconstruction of bone mineral density, which is important in analysis of bone fragility, orthopedic surgery, orthopedic implant design, etc. We hypothesize that full 3D bone anatomy (i.e. bone shape and bone mineral density) for X-ray based diagnosis can be achieved through development of computational and statistical tools, making use of vast amount of femur CT images.

The fundamental objective is to perform multivariate regression on the anatomy of bones. As predictors, the regression model uses patient-specific metadata (e.g. age, weight, body mass index, etc.), and image features extracted from patient radiographs. The variables to predict are patient-specific 3D CT images of bones. As a first step, in this paper we propose univariate parametric regression models based on the exploration of non-parametric regression results for femur bones on a selected subset of clinically meaningful morphological parameters: Femoral shaft length depicted in Fig. 1 $(a, b)$ and caput collum diaphysis (CCD) angle in Fig. 1 (c).

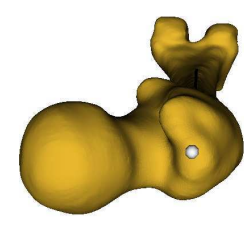

(a)

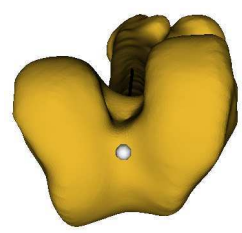

(b)

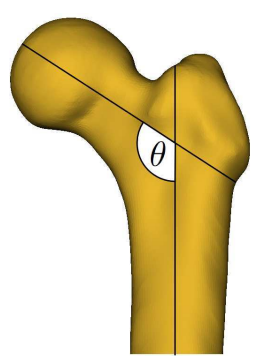

(c)
Fig. 1: (a) Starting point of shaft length morphological parameter at the greater trochanter. (b) End point between condyles. (c) CCD angle.

Currently in medical image regression, parametric (e.g. $[1,2,3])$ and non-parametric (e.g. [4]) approaches have been employed.

- On one hand, parametric-based approaches can be utilized for multivariate regression without encountering the curse of dimensionality problem, but its use preimposes a statistical structure on the data.

- On the other hand, non-parametric approaches do not impose a certain structure, but they suffer from the curse of dimensionality when trying to optimize for hyper-parameters in high-dimensional spaces. However, provided low-dimension subspaces can be found, efficient optimization could be performed. Nevertheless, this is still an open research question $[5,6]$.

Therefore, we selectively combine these two approaches to solve the problem in a low-dimensional space, without preimposing a statistical data structure. For this goal we propose a two-step approach. First, we explore the data structure through univariate non-parametric regression. This step enforces no assumptions on the data structure while avoiding the curse of dimensionality problem. Second, we parametrize the explored structures. 
In [4] the authors showed kernel regression formulated with Fréchet weighted means to take into account the nonEuclidean nature of diffeomorphisms endowed with a right invariant metric (LDDMM) and applied it to images of the brain. In contrast, in this work we formulate kernel regression in an Euclidean way in a Log-Euclidean framework. This simplifies and speeds-up the process significantly while still taking into account a large part of the non-Euclidean nature of the manifold-valued data. Furthermore, this simplification allows for other computations that are out of reach to LDDMM, such as determining the optimal kernel bandwidth through cross-validation. The Log-Euclidean framework uses stationary velocity fields to parametrize a diffeomorphic deformation, whereas in [4] non-stationary velocity fields are used. Although the theory shows that not all diffeomorphic deformation can be reached with stationary velocity fields; there is no indication so far that this affects the anatomical shape analysis in any way.

In the following, we develop the methodology and show results obtained on femur CT images.

\section{METHODS}

The methods description will be divided in five parts: Logdomain registration, Log-Euclidean statistics, non-parametric kernel regression, cross-validation and parametrization of principle component (PC) scores.

\subsection{Log-Domain Registration}

To setup correspondences between anatomical images, a set of images are registered to a reference. We use the novel symmetric diffeomorphic registration approach described in [7]. What is new in this registration framework is the efficient optimization in the log-domain. As a consequence, the results of the registration are so-called stationary velocity fields. These velocity fields can be looked at as generators for diffeomorphic deformations through the group exponential map that can be very efficiently computed using the scaling and squaring method [8].

\subsection{Log-Euclidean Statistics}

Applying the Log-Euclidean framework [8] on these fields allows us to compute statistics, e.g. averages, and still preserve diffeomorphism. In the Log-Euclidean framework, velocity fields are regular elements in a vector space; this allows us to use simple Euclidean statistics instead of more complex non-linear techniques, which we needed when working in the LDDMM space of diffeomorphic transformations. To map resulting velocity fields into diffeomorphic transformations the exponential is calculated. To go from diffeomorphic transformation back to velocity fields, a logarithmic mapping is performed. However, in many cases the intrinsic parametrization of the transformation by its $\log$ in the log-domain registration allows to avoid this numerically unstable step. For a detailed survey of the methodology we refer to [9].

\subsection{Non-Parametric Kernel Regression}

We use a kernel regression method to compute the deformation of the template that best predicts the images based on prediction variables $x$, in our case shaft length and CCD angle. Our kernel regression function is

$$
\hat{m}_{\sigma}(x)=\exp \left(\frac{\sum_{i=1}^{N} K_{\sigma}\left(x-x_{i}\right) v_{i}}{\sum_{i=1}^{N} K_{\sigma}\left(x-x_{i}\right)}\right),
$$

where $N, x_{i}, K_{\sigma}$, exp and $v_{i}$ are the total number of images, shaft length or CCD angle for image $i$, a Gaussian kernel function with $\sigma$ bandwidth, the mapping from velocity fields to diffeomorphic deformations and the $i^{t h}$ velocity field, respectively.

\subsection{Cross-Validation}

The quality of kernel regression methods strongly depends on the selection of bandwidth parameters. To select a bandwidth parameter we apply cross-validation with penalty functions. The penalty and corresponding weighting functions penalize very small bandwidth values. Bandwidth values equal to zero are not interesting because they are just a nearest neighbor interpolation of the data. In our case we solve the following minimization problem:

$$
\hat{\sigma}=\underset{\sigma \in \mathbb{R}}{\operatorname{argmin}} \sum_{i=1}^{N}\left\|\log \left(\hat{m}_{\sigma}\left(x_{i}\right)\right)-v_{i}\right\|^{2} \Xi\left(W_{\sigma, i}\left(x_{i}\right)\right),
$$

where $N, \log , \sigma, \Xi$ are total number of images, mapping from diffeomorphic deformations to velocity fields, bandwidth and penalty function, respectively, and $W_{\sigma, i}\left(x_{i}\right)=$ $K_{1}(0) / \sum_{j=1}^{N} K_{1}\left(\sigma^{-1}\left(x_{i}-x_{j}\right)\right)$ is the weighting function. For details we refer to [10]. By solving this optimization problem we obtain a kernel bandwidth greater than zero which minimizes the regression function's prediction error for all images $N$.

\subsection{Parametrization of Univariate Kernel Regression}

To parametrize the non-linear regression function that we have established via kernel regression, the regressed velocity fields are reduced in dimension using principle component analysis (PCA). The data points are then projected onto a low-dimensional space covering $99 \%$ of the variance and evaluated for possible parametrization. In all cases that we have observed so far it is possible to fit a polynomial to each PC. The procedure can be summarized as follows:

1. Regress velocity fields 


\section{PCA on regressed velocity fields}

3. Plot scores for each PC

4. Fit parametric function to each score plot

The new parameterized regression model is then:

$$
\hat{m}(x)=\exp \left(\mu+\sum_{i=1}^{M} p_{i}(x) z_{i}\right),
$$

where $\mu$ is the mean regressed velocity field, $p_{i}(\cdot)$ is the $i t h$ polynomial function representing ith $\mathrm{PC}$ scores, $z$ are PCs and $M$ the number of PCs describing $99 \%$ of the variance.

Regression is done on 50 predictor values drawn from a normal distribution we obtained on the original data. This way we avoid conducting PCA on the original data and still get a reasonable representation of the original data structure.

\section{RESULTS}

Considering generalized cross-validation (GCV), $\Xi(u)=$ $(1-u)^{-2}$, as the penalty function in (2) we obtain 2.1 for shaft length and 1.6 for CCD angle. With these bandwidth values, prediction is performed on a dataset of 182 left femur CT images.

To validate the results, 50 velocity fields were predicted with prediction values drawn from the normal distribution of the original data. Then the exponential was taken of these fields and the shaft length and CCD angle were measured in the exponentiated fields.

\subsection{Shaft Length Regression}

To find the best parametric model, different polynomials were evaluated. Our evaluation showed that the best fit is linear for all PCs. In Fig. 2 the score plots are depicted and in Tab. 1 four different parametrization and the root mean square (RMS) prediction error are listed. Parametric model 4 performs similar to the non-parametric model.

In Fig. 3 the prediction values are compared in a more qualitative manner.

\begin{tabular}{lllll}
\hline & PC1 & PC2 & PC3 & RMS [mm] \\
\hline Parametric 1 & 1 & 2 & 5 & 1.37 \\
Parametric 2 & 1 & 2 & 3 & 2.26 \\
Parametric 3 & 2 & 2 & 5 & 0.97 \\
Parametric 4 & 1 & 1 & 1 & 0.83 \\
Non-parametric & - & - & - & 0.84 \\
\hline
\end{tabular}

Table 1: Shaft length parametrization comparison. PCn columns show the order of the polynomial fit used for the corresponding PC.

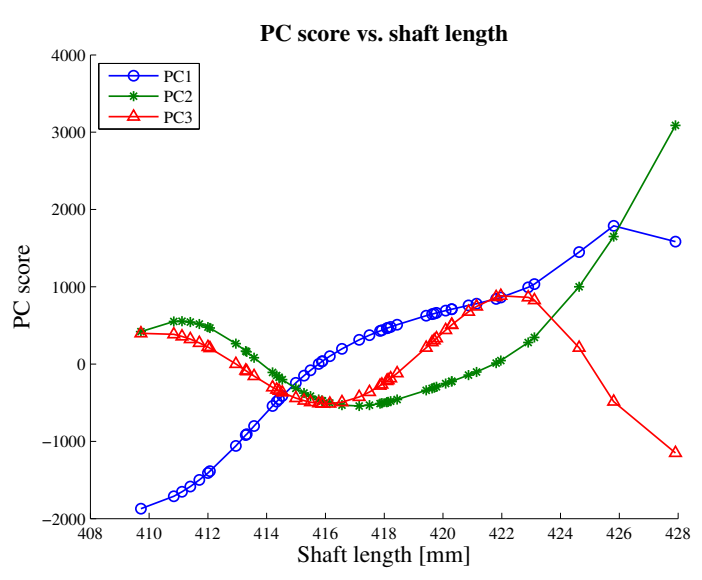

Fig. 2: Score values for the first three PCs.

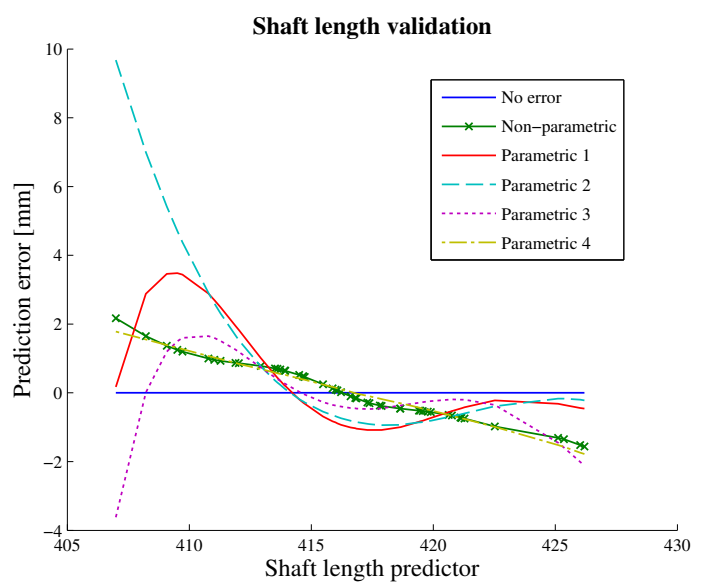

Fig. 3: Comparison of parametric and non-parametric regression results for shaft length.

\subsection{Caput Collum Diaphysis Angle Regression}

Similar to the shaft length low order polynomial give better prediction results. The best prediction results are reached using a linear parametrization for the first PC and quadratic for the second (Tab. 2). This parametric model even outperforms the non-parametric model. See Figs. 4 and 5 for score and prediction error plot.

\section{DISCUSSION AND CONCLUSIONS}

Only three PCs for shaft length and two for CCD angle are needed to describe $99 \%$ of the variance in the velocity fields. This fact enables us to evaluate each predictor individually. Surprisingly, in both experiments low order polynomial parameterizations provided the best results. One possible reason for this could be the behavior of polynomials at the boundary data points. In both cases the parametric slightly outperforms the non-parametric model. It seems that the non-parametric 


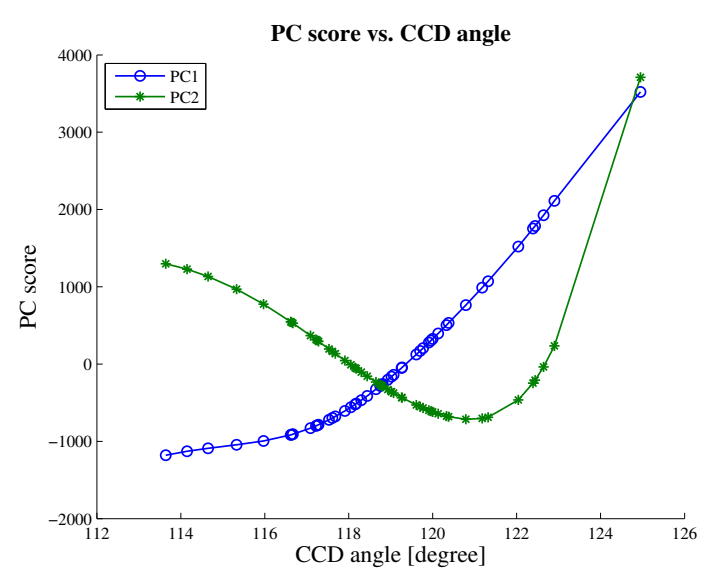

Fig. 4: Score values for the first two PCs.

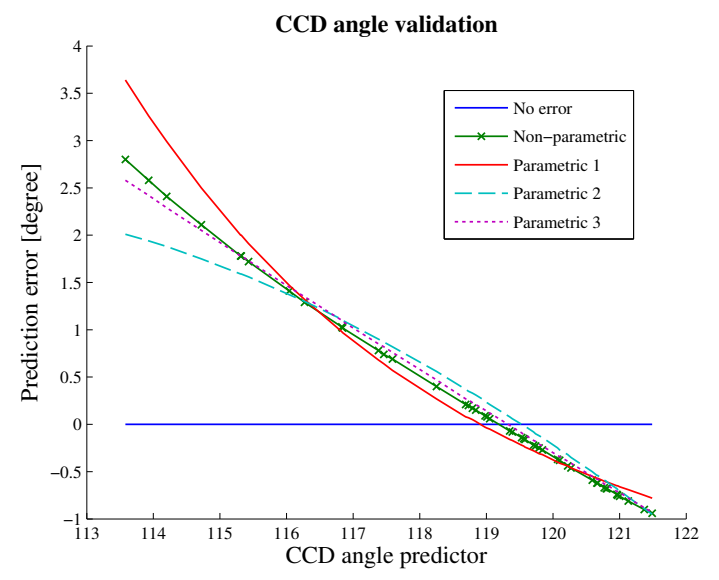

Fig. 5: Comparison of parametric and non-parametric regression results for CCD angle.

model overfits on the training data. Therefore, we gain in three ways from parametric models: Simplicity of the model, computational efficiency and reduction of overfitting.

We plan to evaluate other parametric functions to avoid possible problems at boundary data points. The shown validation can only test the consistency of the method, therefore we will further validate our method by comparing the prediction to manual segmentation results. In future work, simplicity and efficiency, will allow us to use univariate exploration methods to build multivariate regression models with two and more predictors.

\section{REFERENCES}

[1] Yuhui M. Yang, Daniel Rueckert, and Anthony M. J. Bull, "Predicting the Shapes of Bones at a Joint: Application to the Shoulder," Computer Methods in Biomechanics and Biomedical Engineering, vol. 11, no. 1, pp. 19-30, February 2008.

\begin{tabular}{llll}
\hline & PC1 & PC2 & RMS [degrees] \\
\hline Parametric 1 & 2 & 3 & 1.14 \\
Parametric 2 & 1 & 2 & 0.88 \\
Parametric 3 & 1 & 1 & 0.98 \\
Non-parametric & - & - & 1.00 \\
\hline
\end{tabular}

Table 2: CCD parametrization comparison. PCn columns show the order of the polynomial fit used for the corresponding PC.

[2] A. Rao, P. Aljabar, and D. Rueckert, "Hierarchical Statistical Shape Analysis and Prediction of Sub-Cortical Brain Structures," Medical Image Analysis, vol. 12, no. 1, pp. 55-68, February 2008.

[3] T. Liu, D. Shen, and C. Davatzikos, "Predictive Modeling of Anatomic Structures Using Canonical Correlation Analysis," in IEEE International Symposium on Biomedical Imaging: Nano to Macro - ISBI, April 2004, pp. 1279-1282.

[4] B. C. Davis, P. T. Fletcher, E. Bullitt, and S. Joshi, "Population Shape Regression from Random Design Data," in IEEE 11th International Conference on Computer Vision - ICCV, December 2007, pp. 1-7.

[5] S. Gerber, T. Tasdizen, S. Joshi, and R. Whitaker, "On the Manifold Structure of the Space of Brain Images," in Medical Image Computing and Computer-Assisted Intervention - MICCAI, September 2009, pp. 305-312.

[6] F. Steinke and M. Hein, "Non-Parametric Regression Between Riemannian Manifolds," in Conference in Advances in Neural Information Processing Systems, 2009, vol. 21, pp. 1561-1568.

[7] Tom Vercauteren, Xavier Pennec, Aymeric Perchant, and Nicholas Ayache, "Symmetric Log-Domain Diffeomorphic Registration: A Demons-Based Approach," in Medical Image Computing and Computer-Assisted Intervention - MICCAI, 2008, pp. 754-761.

[8] Vincent Arsigny, Olivier Commowick, Xavier Pennec, and Nicholas Ayache, "A Log-Euclidean Framework for Statistics on Diffeomorphisms," in Medical Image Computing and Computer-Assisted Intervention - MICCAI, 2006, pp. 924-931.

[9] Xavier Pennec, "Statistical Computing on Manifolds: From Riemannian Geometry to Computational Anatomy," in Emerging Trends in Visual Computing, vol. 5416 of LNCS, pp. 347-386. Springer, 2008.

[10] Wolfgang Härdle, Applied Nonparametric Regression (Econometric Society Monographs), Cambridge University Press, January 1992. 